\title{
Validazione di un metodo semplice per la stima dell'introduzione di sodio nel paziente in dialisi
}

\author{
P. Cicinato, R. Zanchi, P. Klein, M. Gennari, S. Meot, G. Pacor, P. Rumiz, G. Panzetta \\ Servizio di Nefrologia e Dialisi, Ospedale Maggiore di Trieste
}

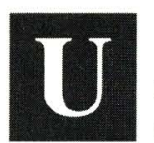

no dei principali compiti della dialisi è l'eliminazione del sodio assunto dai pazienti nell'intervallo interdialitico. Ciò è importante perché variazioni del patrimonio corporeo del sodio determinano modificazioni del volume dei fluidi extracellulari con conseguenze dirette sul sistema cardiocircolatorio dei pazienti.

Il sodio è, infatti, presente pressoché esclusivamente nell'ambiente extracellulare grazie all'azione di pompe del sodio che attivamente estrudono il catione dalle cellule. Una cronica espansione del patrimonio sodico, causando un aumento dell'osmolarità extracellulare, determina un richiamo di acqua dal compartimento intracellulare verso il compartimento extracellulare con espansione del volume circolante e aumento del lavoro del cuore. Al contrario, una riduzione del patrimonio sodico corporeo, diminuendo il potere osmotico extracellulare, determina uno spostamento di acqua dal compartimento extracellulare verso l'intracellulare e favorisce la comparsa di ipotensione, soprattutto durante la dialisi quando il volume extracellulare è ridotto ulteriormente dall'ultrafiltrazione del sangue.

Trascurando gli effetti diretti del sodio sul sistema cardiocircolatorio, quali ad esempio l'aumento della vasocostrizione indotta dall'angiotensina II, la regolazione del patrimonio sodico corporeo deve avvenire con precisione proprio per evitare le modificazioni del volume dei fluidi extracellulari causate dall'elettrolita. Ed infatti il rene naturale aumenta o riduce l'escrezione del sodio non tanto in virtù di modificazioni della sodiemia, ma principalmente sulla base di informazioni sulla grandezza del volume circolante provenienti da sensori dislocati in diversi punti dell'albero cardiocircolatorio, quali gli atri del cuore, l'arco aortico e le arteriole preglomerulari all'interno del rene stesso (1). Il rene naturale è quindi in grado di riportare alla norma rapidamente non solo lo stato di idratazione, ma anche la distribuzione dell'acqua tra i compartimenti fluidi dell'organismo grazie alla sua capacità di eliminare il sodio in quantità variabile rispetto all'acqua totale escreta.

Durante la dialisi, invece, l'eliminazione del sodio è strettamente dipendente dalla quantità di acqua eliminata, dal momento che con ogni litro di plasma ultrafiltrato viene rimossa una quantità di sodio pressoché fissa ed equivalente al contenuto del catione nel litro di plasma ultrafiltrato. Poiché nella maggior parte dei pazienti il sodio e l'acqua vengono introdotti con l'alimentazione secondo un rapporto ben inferiore alla concentrazio- ne del sodio nel plasma, il sodio perso durante la dialisi con un'ultrafiltrazione di plasma pari all'acqua introdotta è quasi sempre superiore alla quantità dell'elettrolita assunto con l'alimentazione.

In generale, quindi, la concentrazione del sodio nel liquido di dialisi è posta a un livello sufficientemente elevato da favorire la diffusione del catione dal bagno al paziente e da compensare la perdita eccessiva determinata dall'ultrafiltrazione pari al calo di peso (2). Più raramente, tuttavia, la concentrazione del sodio nel bagno è posta ad un livello più basso della concentrazione attiva del sodio nel plasma, consentendo così il passaggio dal paziente al bagno e un'ulteriore eliminazione dell'elettrolita.

Idealmente in ogni dialisi dovrebbero essere allontanati tutta l'acqua e tutto il sodio acquisiti dal paziente nell'intervallo interdialitico e per fare ciò sono stati proposti alcuni modelli di cinetica dialitica del sodio capaci di identificare l'opportuna concentrazione sodica del bagno (3-5). Questi modelli hanno dato risultati incostanti e non sono ancora entrati nella pratica clinica. La concentrazione di sodio del bagno viene regolata empiricamente tenendo conto della sodiemia, della pressione arteriosa del paziente e della tolleranza cardiovascolare all'ultrafiltrazione dialitica, cioè sulla base di parame- 
tri che rispecchino piuttosto approssimativamente o per nulla l'entità del patrimonio sodico dell'organismo.

La conoscenza della quantità di sodio introdotta dai pazienti costituirebbe, invece, un utile mezzo per migliorare le decisioni operative (6). Essa consentirebbe infatti di distinguere se alcuni segni clinici di un eccesso o di una deplezione di sodio siano più probabilmente frutto di un'alterazione dell'introito dietetico o dell'uso di un bagno di dialisi incongruo portando così a correzioni della dieta $o$ invece della concentrazione sodica del bagno.

È stato suggerito che l'introduzione di sodio dei pazienti può essere stimata dall'incremento del patrimonio sodico corporeo dalla fine della dialisi all'inizio della dialisi successiva (7). Per calcolare ciò è necessario disporre delle sodiemie post e predialitiche e dei volumi di acqua corporea totale misurati agli stessi tempi. Nonostante questo metodo di calcolo delle variazioni del patrimonio corporeo del sodio sia ritenuto attendibile in diversi contesti clinici e nonostante l'evidente utilità di questo parametro, non vi sono lavori che si siano proposti di verificarne l'attendibilità nei pazienti in dialisi e il suo impiego ripetitivo nella pratica clinica.

Scopo del presente lavoro è stato quello di calcolare l'apporto sodico con questo metodo e di confrontarlo con l'escrezione del sodio misurata durante la seduta depurativa: una corrispondenza dei due dati sarebbe infatti indicativa dell'attendibilità del metodo stesso. Per aumentare la precisione dell'indagine abbiamo esteso la valutazione a tre trattamenti consecutivi ed abbiamo utilizzato l'emofiltrazione che meglio consente di eseguire studi di bilancio del sodio grazie ai più bassi volumi di fluidi utilizzati.

\section{Materiali e metodi}

Sono stati studiati 9 pazienti ( $5 \mathrm{M}$ e $4 \mathrm{~F}$ ) di età compresa tra 51 e 86 anni (media 69.4) in trattamento con emofiltrazione da $20.7 \pm 18.5$ mesi. I pazienti erano clinicamente stabili ed esenti da malattie che potessero ostacolare una corretta alimentazione o determinare una perdita corporea di elettroliti. Nessuna istruzione dietetica particolare è stata data ai pazienti nel corso dello studio che, d'altro canto, non ha comportato alcuna interfe- renza sulla loro normale vita di relazione. La maggior parte dei pazienti era virtualmente anurica e solo in due casi la dieuresi era intorno a $300 \mathrm{ml}$ al giorno.

\section{Tecnica dell'emofiltrazione}

I pazienti erano sottoposti alla loro normale seduta di emofiltrazione di circa 4 ore con filtri in Poliamide, con ultrafiltrazione pari a $30.9 \pm 2.7 \mathrm{~L}$ e con reinfusione in postdiluizione pari a $27.7 \pm$ $2.6 \mathrm{~L}$.

\section{Metodi di indagine}

Lo studio iniziava al termine dell'ultima emofiltrazione della settimana e si concludeva al termine della terza emofiltrazione della settimana successiva. All'inizio ed alla fine di ogni seduta venivano prelevati dalla fistola $2.5 \mathrm{ml}$ di sangue eprinizzato per dosare la sodiemia in triplicato con fotometro a fiamma (Ciba Corning 480).

Prima dell'inizio della seconda dialisi della settimana veniva misurata l'acqua totale corporea mediante bioimpedenziometro (BIA 109 Akern): il volume dell'acqua corporea alla fine ed all'inizio di ogni seduta veniva stimato aggiungendo o sottraendo al dato misurato con il bioimpedenziometro la variazione registrata del peso corporeo.

Il sodio introdotto dai pazienti in ognuno dei tre intervalli era calcolato con la formula ( $\mathrm{CtVt}$ - COV0), dove $\mathrm{Ct}$ e $\mathrm{C} 0$ sono le concentrazioni plasmatiche del sodio alla fine dell'emofiltrazione e all'inizio dell'emofiltrazione successiva, e Vt e V0 sono i volumi di acqua corporea totale stimati agli stessi momenti.

Il sodio eliminato dai pazienti durante ognuno dei tre trattamenti veniva calcolato come differenza tra il sodio perso nell'ultrafiltrato ed il sodio guadagnato con l'infusato, con la soluzione fisiologica di restituzione e con ogni eventuale liquido contenente sodio somministrato durante la seduta (dosaggi in triplicato con fotometro a fiamma).

\section{Risultati}

La Tabella I mostra il valore medio e la deviazione standard di ogni parametro esaminato durante $i$ tre intervalli e $i$ tre trattamenti in ognuno dei 9 pazienti.

È possibile notare che nell'insieme dei 9 pazienti la concentrazione plasmatica del sodio passava da $141.2 \pm 1.7 \mathrm{mEq} / \mathrm{L}$ a $140 \pm 2.1 \mathrm{mEq} / \mathrm{L}$ dalla fine delle emofiltrazioni all'inizio delle successive per risalire nuovamente a $141.2 \pm 1.8 \mathrm{mEq} / \mathrm{L}$ nel corso delle stesse.

Il volume di acqua totale corporea, misurato agli stessi tempi variava da $32.4 \pm$ $6.8 \mathrm{~L}$ a $35.1 \pm 7.1 \mathrm{~L}$, per ridiscendere a $32.4 \pm 6.8 \mathrm{~L}$ al termine delle emofiltrazioni.

L'introduzione di sodio dei pazienti, calcolata sulla base dei dati post e predialitici, risultava pari a $338 \pm 66 \mathrm{mEq}$ per un intervallo di 3120 minuti (media di un intervallo lungo e di 2 corti), cioè pari a $1014 \mathrm{mEq}$ per settimana o $145 \mathrm{mEq}$ al giorno.

La quantità media di ultrafiltrato e di infusato delle tre sedute e le relative concentrazioni sodiche sono presentate nella Tabella II. In questa tabella è anche evidenziata la quantità di sodio somministrato con la fisiologica e con gli altri liquidi contenenti sodio. L'eliminazione media di sodio risultava $329 \pm 67 \mathrm{mEq}$ per seduta, pari a $987 \mathrm{mEq}$ la settimana. Vi era quindi stretta corrispondenza tra il calcolo del sodio mediamente introdotto dai pazienti e la misura del sodio eliminato durante le sedute: $338 \mathrm{mEq}$ e 329 $\mathrm{mEq}$.

La Figura 1 mostra la relazione altamente significativa esistente tra il sodio mediamente introdotto e quello eliminato dai nove pazienti esaminati $(r=0.92 ; \mathrm{p}<$ 0.0001 ). Ulteriori relazioni significative sono risultate tra introito calcolato di sodio e incremento ponderale $(\mathrm{r}=0.89 ; \mathrm{p}<$ 0.001 ) (Fig. 2), così come tra eliminazione misurata di sodio e calo ponderale medio durante la seduta $(r=0.88 ; \mathrm{p}<$ 0.001 ) (Fig. 3).

Nessuna correlazione veniva invece riscontrata tra la taglia dei pazienti, identificata dal peso corporeo o dall'acqua totale, e l'introduzione di sodio.

Quando venivano esaminate le corrispondenze tra l'introduzione e l'eliminazione di sodio in ogni singolo intervallo e in ogni successiva emofiltrazione, la significativa statistica della relazione tra $i$ due parametri era ancora elevata $(\mathrm{r}=0.50$; $\mathrm{p}<0.01$ ), ma la dispersione dei dati aumentava in confronto alla quasi precisa identità dei valori medi dei tre intervalli 
TABELLA I - VALORE MEDIO DELLA SODIEMIA E DEL VOLUME DI ACQUA CORPOREA MISURATI IN OGNI PAZIENTE ALLA FINE DI TRE SEDUTE (POST 1), ALL'INIZIO (PRE 2) E ALLA FINE (POST 2) DELLE TRE SEDUTE SUCCESSIVE. L'INTROITO DI SODIO È QUELLO MEDIO. MISURATO DALLA FINE DELLE TRE SEDUTE ALL'INIZIO DELLE TRE SEDUTE SUCCESSIVE

\begin{tabular}{lcccccccccc}
\hline N. paziente & 1 & 2 & 3 & 4 & 5 & 6 & 7 & 8 & 9 & Media \pm DS \\
\hline Sodiemia post1 (mEq/L) & 142.8 & 141.5 & 144.1 & 142.3 & 140.1 & 141.2 & 139.2 & 139.5 & 139.6 & $141.2 \pm 1.7$ \\
Sodiemia pre2 (mEq/L) & 141.5 & 140.2 & 143.8 & 142 & 138.3 & 139.1 & 138 & 137.2 & 140.3 & $140 \pm 2.1$ \\
Sodiemia post2 (mEq/L) & 143.5 & 140.1 & 144.5 & 141.2 & 140.5 & 141.3 & 138.7 & 140.5 & 140.1 & $141.2 \pm 1.8$ \\
Acqua corporea post1 (I) & 20.9 & 43.4 & 35 & 28.6 & 34.8 & 38 & 35.7 & 26 & 29.2 & $32.4 \pm 6.8$ \\
Acqua corporea pre2 (I) & 22.8 & 46.1 & 37.3 & 31.8 & 37.7 & 41.4 & 38.2 & 28.2 & 32.3 & $35.1 \pm 7.1$ \\
Acqua corporea post2 (I) & 20.9 & 43.3 & 35 & 28.7 & 34.6 & 38.4 & 35.6 & 26 & 29.2 & $32.4 \pm 6.8$ \\
Introito di sodio (mEq) & 238 & 324 & 319 & 435 & 342 & 390 & 293 & 250 & 454 & $338 \pm 75$ \\
\hline
\end{tabular}

TABELLA II - VALORE MEDIO DI TRE SEDUTE DI OGNI PAZIENTE PER QUANTITÀ DI ULTRAFILTRATO (Q-UF), CONCENTRAZIONE DI SODIO NELL'ULTRAFILTRATO (NA-UF), QUANTITÀ DI INFUSATO (Q-INF), CONCENTRAZIONE DI SODIO NELL'INFUSATO (NA-INF), SUPPLEMENTO DI SODIO FORNITO AI PAZIENTI. LA QUANTITÀ DI SODIO ELIMINATO (Q-NA) È QUELLA MEDIA DELLE TRE SEDUTE

\begin{tabular}{lcccccccccc}
\hline N. paziente & 1 & \multicolumn{1}{c}{2} & \multicolumn{1}{c}{3} & \multicolumn{1}{c}{4} & \multicolumn{1}{c}{5} & 6 & 7 & \multicolumn{1}{c}{8} & 9 & Media \pm DS \\
\hline Q-UF (I) & 26.5 & 32.6 & 34 & 30.4 & 32 & 32.9 & 31.9 & 26.3 & 31.9 & $30.9 \pm 2.7$ \\
Na-UF (mEq/L) & 140.6 & 139.7 & 140.9 & 140.4 & 138.3 & 139.7 & 137.9 & 138.8 & 139.5 & $139.5 \pm 1$ \\
Q-Inf (I) & 24 & 29.2 & 31.3 & 26.9 & 27.6 & 29.6 & 29 & 23.5 & 28.3 & $27.7 \pm 2.6$ \\
Na-Inf (mEq/L) & 143.3 & 142.2 & 143 & 141.5 & 141.5 & 141.8 & 140.5 & 141.5 & 142.2 & $141.9 \pm 0.8$ \\
Na-Suppl (mEq) & 76.5 & 44.6 & 33.2 & 44.2 & 30.6 & 30.7 & 30.7 & 55.6 & 27.5 & $41.5 \pm 16$ \\
Q-Na (mEq) & 204 & 350 & 336 & 415 & 350 & 349 & 287 & 268 & 399 & $329 \pm 67$ \\
\hline
\end{tabular}

e delle tre emofiltrazioni (Fig. 4). Ciò dimostra che ogni seduta era lontana dall'eliminare esattamente la quantità introdotta di sodio e che erano necessarie le tre sedute della settimana per ristabilire il bilancio sia dei liquidi che del sodio introdotto nello stesso arco di tempo.

\section{Discussione}

Lo studio ha dimostrato un'ottima correlazione tra la quantità di sodio eliminata dai pazienti con le tre emofiltrazioni e la quantità di sodio introdotta nello stesso periodo di tempo. Questo dato conferma la possibilità di stimare con precisione l'introito dietetico di sodio ricorrendo al- la semplice misurazione del sodio plasmatico e dell'acqua corporea totale alla fine di un trattamento e all'inizio del successivo.

Lo studio ha anche confermato che l'eliminazione del sodio durante una singola seduta non corrisponde esattamente alla quantità introdotta nell'intervallo precedente, poiché, come è noto, la cinetica di eliminazione del sodio è influenzata da molte variabili, quali la differenza della concentrazione del sodio tra ultrafiltrato ed infusato e soprattutto la quantità di ultrafiltrato corrispondente al calo di peso del paziente. Questi elementi possono variare cospicuamente da trattamento a trattamento senza alcuna relazione con l'introito di sodio dei pazienti. Nell'arco della settimana, tuttavia, il bilancio sodico risulta in pareggio, come è logico attendersi in una condizione fisiopatologica che per definizione prevede l'equilibrio tra la quantità introdotta o generata di una sostanza e la quantità eliminata dall'organismo. Ciò che può essere alterato è la grandezza del patrimonio corporeo della sostanza, che può restare aumentato o ridotto anche quando, come nel caso del sodio, speciali modelli cinetici di eliminazione realizzino da un certo punto in poi la perfetta corrispondenza tra le entrate e le uscite in ogni trattamento.

Poiché non esistono mezzi clinicamente utilizzabili che consentano di stimare l'adeguatezza del patrimonio di sodio in un 


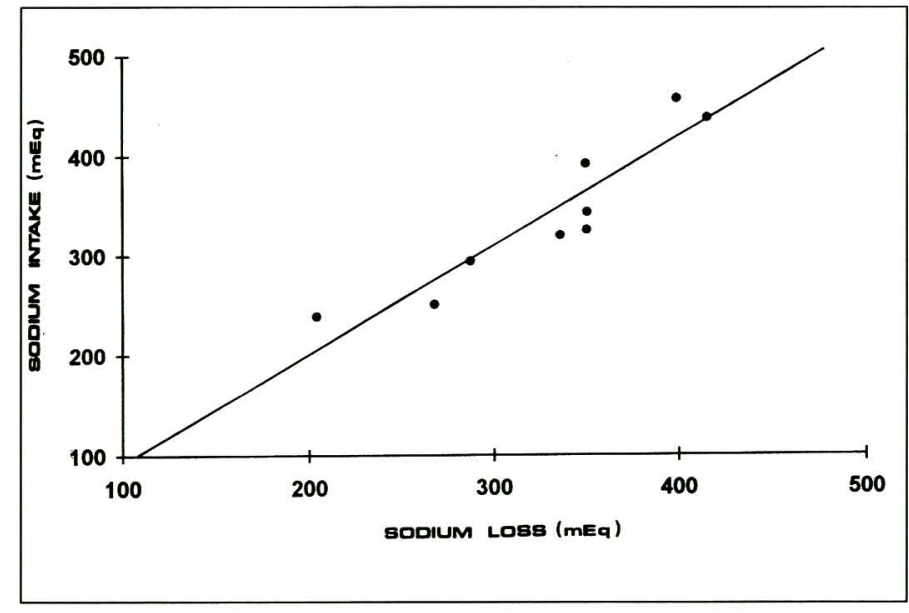

Fig. 1 - Relazione diretta $(r=0.92 ; p<0.0001)$ tra sodio mediamente introdotto nell' intervallo e sodio eliminato durante le sedute nei 9 pazienti.

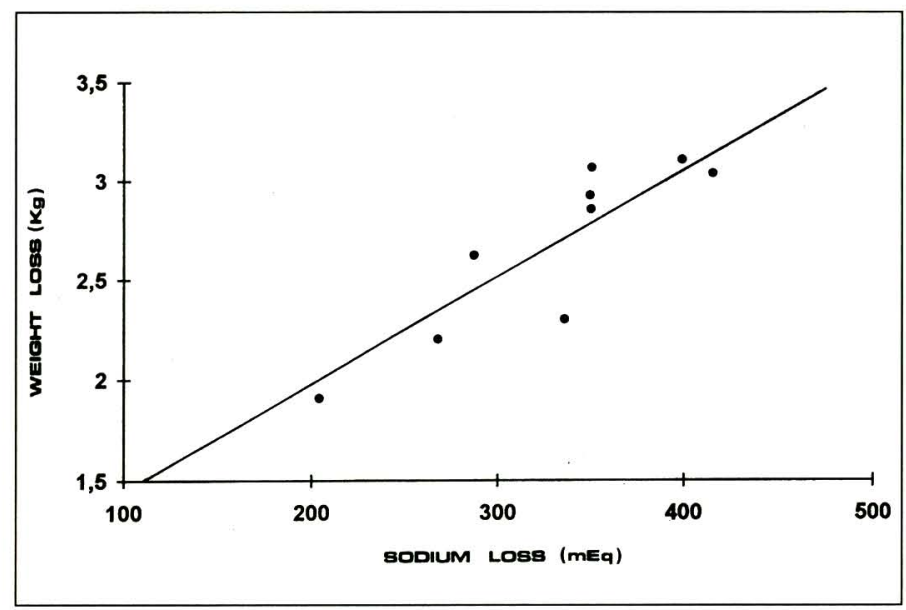

Fig. 3 - Relazione diretta $(r=0.88 ; p<0.001)$ tra eliminazione media di sodio e calo ponderale durante le sedute nei 9 pazienti.

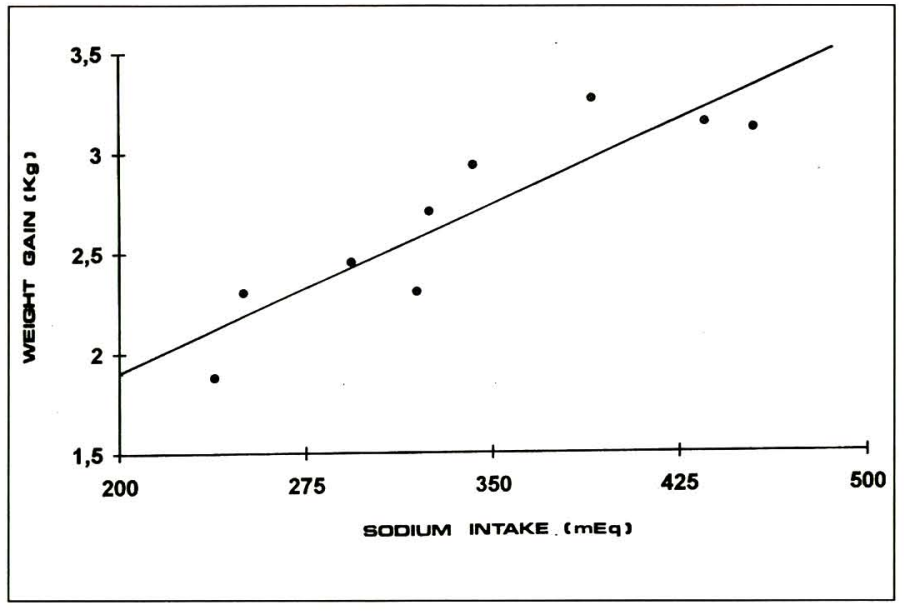

Fig. 2 - Relazione diretta $(r=0.88 ; p<0.001)$ tra introito medio di sodio e incremento ponderale nei 9 pazienti.

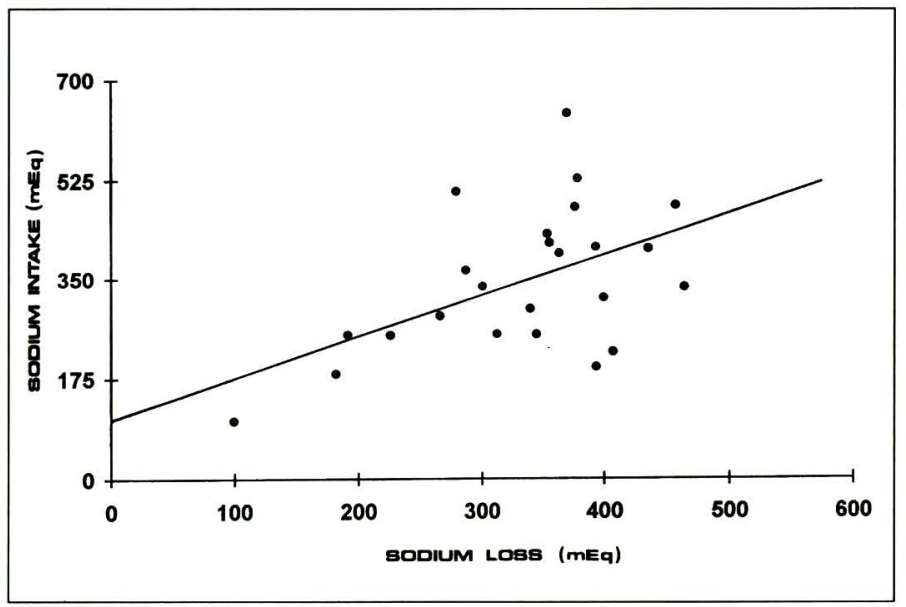

Fig. 4 - Relazione diretta $(r=0.50 ; p<0.01)$ tra eliminazione di sodio di ogni seduta e introito di sodio dell' intervallo precedente. soggetto in dialisi, la scelta della concentrazione del sodio nel bagno resta empirica. Tuttavia, la conoscenza della quantità di sodio introdotto dal paziente può ridurre i margini di incertezza.

È ben noto, ad esempio, che l'aumento della concentrazione del sodio nel bagno migliora la tolleranza alla dialisi e riduce il numero delle ipotensioni $(8,9)$. Ciò può esporre, però, al rischio di espansione patologica del patrimonio sodico dei pazienti con conseguente sovraccarico del circolo (10). Particolarmente quando si trovino valori di sodiemia predialitica ai limiti superiori della norma o già sopra la norma, potrà essere opportuno ridurre un apporto dietetico di sodio rile- vatosi elevato o sarà necessario ridurre l'alta concentrazione sodica nel bagno quando l'introito sodico risulterà normale o basso. Nel primo caso potranno permanere gli effetti utili del bagno e contemporaneamente sarà evitato il sovraccarico corporeo di sodio.

All'opposto non è raro il riscontro di pazienti con iposodiemia, che trova generalmente spiegazione in un apporto alimentare modesto di nutrienti e di sale e in una deplezione sodica indotta dall'ultrafiltrazione per incrementi di peso non contenuti proporzionalmente. Il rilievo di un basso apporto dietetico di sodio potrà indurre ad incoraggiare l'introito di sale in questi pazienti o convincerà dell'op- portunità di utilizzare bagni ipersodici e/o supplementi di cloruro di sodio endovena durante la seduta. Simili provvedimenti possono migliorare la tolleranza cardiovascolare all'ultrafiltrazione e possono eventualmente consentire di correggere uno stato di iperidratazione spesso presente nei pazienti sodiodepleti a causa dell'alta frequenza di ipotensioni dialitiche.

Nel nostro studio abbiamo rilevato una stretta correlazione diretta tra calo di peso dei pazienti ed eliminazione di sodio durante la seduta e anche un'altrettanto stretta correlazione diretta tra l'introito di sodio e la crescita di peso dei pazienti. Questi dati possono presentarsi ad una 
duplice interpretazione: è possibile che $\mathrm{i}$ pazienti introducano proporzionalmente tutte le sostanze alimentari, sodio e acqua compresi, oppure vi è una stimolazione della sete e dell'introduzione dell'acqua dipendente dalla quantità del sale introdotto. Sebbene la corretta interpretazione dei fenomeni non sia agevole, la mancanza di correlazione tra l'introduzione di sodio o di acqua da un lato e la taglia corporea dei pazienti dall'altro, rendono più probabile l'ipotesi che $\mathrm{i}$ pazienti siano indotti a bere più acqua quando l'introduzione di sale sia più alta. Se questi dati saranno confermati in un numero più grande di pazienti, risulterà chiara l'indicazione a controllare regolarmente l'apporto sodico (per cercare di limitarlo, se necessario) quando si riscontrino incrementi di peso eccessivi. Conviene precisare, in questo contesto, che non abbiamo riscontrato alcuna relazione tra l'aumento di peso dei pazienti e la sodiemia misurata sia alla fine sia all'inizio della seduta.

Come corollario a questo lavoro è opportuno segnalare che non abbiamo trovato differenze significative nel calcolo dell'introduzione di sodio usando per stima dell'acqua corporea la formula antropometrica di Watson (11) invece della bioimpedenziometria (338 $\pm 66 \mathrm{mEq}$ vs $339 \pm 78 \mathrm{mEq}$ di sodio introdotto per intervallo). La corrispondenza dei risultati con i due metodi deriva dalla prevalente importanza nei calcoli del cambiamento del volume dell' acqua corporea totale durante l'intervallo (perfettamente identificabile con l'aumento del peso corporeo) rispetto al valore di partenza dell'acqua corporea.

Quest'ulteriore semplificazione nel metodo, che evita la stima precisa dell'acqua corporea, consentirà un'ampia applicazione clinica della misura dell'apporto sodico nei pazienti dializzati e la rapida valutazione dell'utilità di questo parametro.

\section{BIBLIOGRAFIA}

1. Kirchner KA, Stein JK. Sodium metabolism. In: Maxwell and Kleeman's Clinical disorders of fluid and electrolyte metabolism (Fifth Edition). Mc Grow-Hill, Inc, 1994; 45-80.

2. Kirmurag G, Van Stone JC, Baver J, Keshaviah P. A simulation study on transcellular fluid shifts induced by hemodialysis. Kidney Int 1983; 24: 542-8.

3. Gotch FA, Lam FA, Prowitt M, Keen ML. Preliminary clinical results with sodium volume modeling of hemodialysis therapy. Proc Clin Dial Transplant Forum 1980; 10: 12-6.

4. Petitclerc T, Hamani A, Jacobs C. Optimization of sodium balance during hemodialysis by rotuine implementation of kinetic modeling: Technical aspects and preliminary clinical study. Blood Purif 1992; 10 : 309-16.

5. Pepticlerc T, Coevoet B, Trombert JC, Jacobs C. Prevention of intradialytic morbility by routine implementation of sodium modeling. Blood Purif 1994; 12: 163-4. (abstract).

6. Van Stone JC. Individualization of the dialysate prescription in chronic hemodialysis. Dial Transplant 1994; 23: 624-35.

7. Sargent JA, Gotch FA. Principles and biophysics of dialysis. In: Maher JF, ed. Replacement of renal function by dialysis (Third edition, 1989). Kluwer Academic Publishers 87-143.

8. Wehle B, Asaba H, Castenfors $\mathrm{J}$, et al. The influence of dialysis fluid composition on the blood pressure response during dialysis. Clin Nephrol 1978; 10: 62.

9. Van Stone JC, Cook J. Decrea- sed postdialysis fatigue with increased dialysate sodium concentration. Proc Clin Dial Transplant. Forum 1978; 8: 152.

10. Robson R, Oren A, Ravid M. Dialysate sodium concentration, hypertension and pulmonary edema in hemodialysis patients. Dial Transplant 1978; 7: 678-9. 